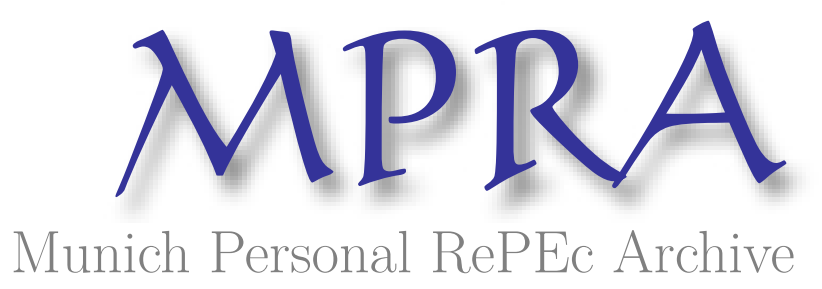

\title{
Decomposing Altruism - An Experiment to Measure Motivations for Giving by Demographic Group
}

Mayo, Robert

23 March 2017

Online at https://mpra.ub.uni-muenchen.de/98431/

MPRA Paper No. 98431, posted 01 Feb 2020 11:11 UTC 


\title{
Decomposing Altruism:
}

\section{An Experiment to Measure Motivations for Giving by Demographic Group.}

\author{
Robert L. Mayo ${ }^{\dagger}$
}

March 23, 2017

\begin{abstract}
I conduct an on-line experiment to decompose giving in a dictator game into amounts motivated by pure altruism and amounts motivated by the warm glow of giving as defined in Andreoni (1989). By manipulating the price of benefit to the recipient while holding the price of the act of giving constant, I estimate separate values for giving motivated by warm glow and giving motivated by pure altruism,. I find significant evidence of both pure altruism and warm glow as motivations for the amounts sent to an anonymous recipient. However, I also find a large gender difference in the motivation for giving. Females are significantly less sensitive to the price of benefit to the recipient than are males, suggesting females are motivated relatively more by warm glow and relatively less by pure altruism, while men display the opposite behavior.
\end{abstract}

JEL Classification: C99, D31, D64

Keywords: Warm glow; Charitable giving; Altruism; Gender

$\dagger$ Department of Economics, George Mason University, rmayo3@gmu.edu. I wish to acknowledge, with gratitude, Dr. Kevin McCabe for his advice and the Center for the Study of Neuroeconomics for generously providing funding to support this research. All errors or omissions are the sole responsibility of the author. 
"How selfish soever man may be supposed, there are evidently some principles in his nature which interest him in the fortune of others and render their happiness necessary to him though he derives nothing from it except the pleasure of seeing it"

Adam Smith, The Theory of Moral Sentiments

"It is not from the benevolence of the butcher, the brewer, or the baker, that we can expect our dinner, but from their regard to their own interest"

Adam Smith, The Wealth of Nations

\section{Introduction}

In this paper, I use experimental data from a dictator game conducted over the internet to decompose giving into its altruistic and egoistic components in a within subject design, thus allowing a measurement of the warm glow effect (Andreoni 1989). I find giving motivated by warm glow and giving motivated by pure altruism across demographic groups based on selfreported responses to an exit survey, which allows a comparison of motivations in giving based on age, gender, and income.

In economic experiments, subjects routinely choose not to maximize their payoffs. In prisoners' dilemmas, subjects frequently cooperate (Doebeli and Hauert 2005). In public goods games, subjects often contribute (Zelmer 2003). The simplest example of this behavior is seen in the dictator game. In the dictator game, one subject is given an amount of money and then allowed to send any portion of that amount to another subject. The Nash equilibrium is to send nothing. While it is true that many subjects do keep all the money, many do not. A significant fraction of subjects sends positive amounts (Engel 2011). Traditional, neo-classical economic theory presents man as a rational, maximizing agent, motivated solely by material rewards to self. This interpretation of human action does model economic behavior well in many circumstances. However, in addition to failing to account for a wide variety of results in economic experiments, it does not explain large portions of economic activity seen in the real world.

Addressing this gap in our understanding, Andreoni (1989) developed an impure altruism model of giving that included two distinct patterns of other-regarding behavior. The paper 
proposed that giving could be motivated by pure altruism where the giver derives utility from the benefit the gift provides to the recipient, and by pure egoism where the giver derives utility from the act of giving itself independent of its impact on the recipient; calling this latter effect warm glow. A mixture of warm glow and pure altruism as motivation is characterized as impure altruism. That paper also proposed an index of pure altruism $\alpha_{i}$ such that:

$$
\alpha_{i}=\frac{f_{i a}}{f_{i a}+f_{i e}}
$$

Where $f_{i a}$ is the amount given by person $i$ that is motivated by pure altruism and $f_{i e}$ is the amount given by person $i$ that is motivated by warm glow (Ibid., p.1452).

Several prior studies have tested for the existence of warm glow and pure altruism as motivations for giving (Ribar and Wilhelm 2002), (Gangadharan, Grossman, and Jones 2014), but few have attempted to quantify the effect and to the author's knowledge none have done so by demographic group. Research addressing warm glow frequently takes the perspective of giving in a public goods context. An example of this is Palfrey and Prisbrey (1997) which uses a public goods game with variable private values. They find evidence of a small warm glow effect, but no significant evidence of pure altruism. By contrast and also in the context of a public goods game, Goeree, Holt, and Laury (2002) find statistically significant evidence of altruism but reject warm glow as a causal factor in subject behavior. Crumpler and Grossman (2008) use a simple and innovative design, also used in (Luccasen and Grossman 2017), where subjects can give to a charity, but with a perfect crowding out effect. Any amount given by the subject reduced by an equal amount a donation given to the charity by the experimenter. Knowing that their contributions would not alter the amount received by the charity, 57\% of subjects still made a positive contribution. Across all subjects, contributions averaged $20 \%$ of their endowments which is strong evidence of warm glow as a motivation. Lilley and Slonim (2014) examined warm glow giving in a study of the volunteering puzzle, where people donate time to charities even when donating the wage equivalent sum of money is more efficient. They find evidence of a mixture of pure altruism and warm glow as motivating factors in charitable contribution and estimate between $15.5 \%$ and $21 \%$ of amounts donated were motivated by warm glow. In a study of choice between volunteering time or donating money, Brown, Meer, and Williams (2013) find that subjects prefer to donate time rather than money even when it is the 
less efficient choice. They argue that the cause is a preference for the warm glow of volunteering. Tonin and Vlassopoulos (2014) decompose charitable giving by motivation and find significant evidence of both war glow and pure altruism. Konow (2010) find that charitable giving cannot be explained by warm glow alone and is more consistent with subjects following context specific economic norms. Karlan and List (2005) use a natural field experiment of charitable giving and find that providing matching funds increases amount donated, but higher match rates from $\$ 1: \$ 1$ to $\$ 3: \$ 1$ do not significantly increase donations further. Eckel and Grossman (2003) find framing an additional amount as a match rather than as a mathematically equivalent rebate increases amounts given. Tonin and Vlassopoulos (2010) find a demographic difference in giving motivation with warm glow being a significant factor for women but not for men, and for neither gender do they find evidence of pure altruism. In this paper, I extend the literature by measuring differences in the relative influence of warm glow and pure altruism as motivations for giving across demographic groups of age, income, and gender.

The remainder of this paper is organized as follows. The next section presents the theoretical model of giving. Section 3 describes the experimental design. Section 4 reviews results. Section 5 is discussion, and section 6 concludes.

\section{Model}

I differentiate between giving motivated by benefit to the recipient and giving motivated by benefit to the giver by varying their relative prices. If I observe dictators sending the same amount of money regardless of how much I as experimenter multiply it, then I can conclude that they are motivated by the constant price of the act of giving and not by the varying price of benefit to the recipient.

Assume a dictator $i$ has an endowment $y_{i}$ and utility function $u_{i}=f\left(x_{i}, z_{i}, z_{j}\right)$ where $x_{i} \in\left[X \mid 0 \leq x_{i} \leq y_{i}\right]$ is the portion of $y_{i}$ retained, $z_{i} \in\left[Z \mid z_{i}=y_{i}-x_{i}\right]$ is the amount sent to another subject $j$, and given multiplication factor $\mu, z_{j} \in\left[Z \mid z_{j}=\mu z_{i}\right]$ is the amount received by subject $j$. Also assume $\mu=1$. The choice of $z_{i}$ may be motivated by the welfare of the recipient entering directly into the utility function of the dictator $z_{i}$; utility from the act of giving unrelated to the welfare of the recipient $z_{j}$; or a mixture of the two motives, balanced against the amount retained $x_{i}$. Now, assume that for an amount $z_{i}$ sent by the same dictator, an anonymous 
recipient will receive $z_{j}=\mu z_{i}$ where $\mu>1$. If the dictator is motivated by $x_{i}$ and $z_{j}$, then the dictator will reallocate towards $z_{j}$ and away from $x_{i}$ compared to the prior case where $\mu=1$. However, if the dictator receives utility from $z_{i}$ but not from $z_{j}$ then no reallocation will occur. By observing the extent to which subjects vary $z_{i}$ as a function of $\mu$, I can infer the relative contributions to utility from both $z_{i}$ and $z_{j}$, and estimate values for Andreoni's $\alpha$ which is the ratio of giving due to pure altruism to total giving.

I implement this analysis by asking subjects how much of an initial endowment they would chose to send to an anonymous, passive counterpart given that the amount chosen will be multiplied by the experimentor. Each subject is presented with a variety of values for the multiplier $\mu \in\{0.2,0.4,0.6,0.8,1,2,3,4,5\}$. At $\mu=0.2$ the counterpart receives $20 \%$ of the amount sent, up to $\mu=5.0$ where the counterpart receives $500 \%$ of the amount sent, with one choice randomly selected for payment. Using a continuous range from 0.2 to 5.0 was considered (List, Sadoff, and Wagner 2011), but was not chosen in order to reduce subjects computaional burdon.

Given that the amounts subjects choose to send are censored from below by zero and from above by the $\$ 1$ endowment, OLS estimates of the conditional mean would be biased (Rigobon and Stoker 2009). To avoid this bias, I use quantile regression of the conditional median. I chose this option because it is robust to censoring (Portnoy 2016) and non-parametric, thus avioding the both the sensitivity to heteroskedasticity found in Tobit estimation (Arabmazar and Schmidt 1982) and the necessesity of assumptions about the data generating process ${ }^{1}$.

For each subject's set of choices of amounts to send, I perform the regression shown in equation 2 , where $x_{i}$ is the amount retained and $\mu$ is the value of the multiplier. The initial endowment is $\$ 1.00$, so $1-x_{i}$ is the amount sent by dictator $i$.

$$
\left(1-x_{i}\right)=\hat{\beta}_{0}+\hat{\beta}_{1} \mu+\epsilon_{i}
$$

The estimate of the amount sent that does not vary as a function of the multiplication factor is the constant $\hat{\beta}_{0}$. This is the portion of $1-x_{i}$ that was motivated by factors unrelated to

${ }^{1}$ The appearance of a large cluster of zero values can be consistent with non-censored data if the data is generated by two processes; one determining whether the value will be zero or non-zero, and another process determining the value given that it is not zero. For a further discussion, see Greene (2008) pp. 854-857. 
the benefit of the recipient and so is the isolated influence of warm glow. $\hat{\beta}_{1} \mu$ is the estimate of the amount sent that was motivated by the variable benefit of the recipient, and therefore is the isolated influence of pure altruism. And so, $\hat{\beta}_{0}+\hat{\beta}_{1} \mu$ is the estimate of the total amount sent prompted by both motivations. Substituting these values into equation 1 gives equation 3

$$
\hat{\alpha}_{i}=\frac{\hat{\beta}_{1}}{\hat{\beta}_{0}+\hat{\beta}_{1} \mu}
$$

This calculation raises two issues. First, since subjects are presented with a range of values for $\mu$, if they are motivated by pure altruism to any extent (i.e. $\hat{\beta}_{1} \neq 0$ ) then $\hat{\alpha}_{i}$ will not be constant across $\mu$. Therefore, to facilitate comparisons with other studies that may not include a multiplier, I base all estimates of $\alpha$ on a value of $\mu=1$ A second issue is how to code $\hat{\alpha}$ when both $\hat{\beta}_{0}$ and $\hat{\beta}_{1}$ equal zero. Since this is the case when a dictator always sends nothing regardless of value of $\mu$, I define $\left(\hat{\beta}_{0}=\hat{\beta}_{1}=0\right) \Rightarrow(\hat{\alpha}=0)$ on the basis that since nothing was given, pure altruism could not be a motivation for giving. It should be noted that in the case where a subject always gives nothing, the absence of pure altruism does not imply the existence of warm glow.

After decomposing dictator giving by motivation for each subject, I test two sets of hypotheses:

First, I test whether warm glow and pure altruism exist. Hypotheses 1a and 1b: Warm glow and pure altruism respectively are significant motivators for giving.

$$
\begin{aligned}
& H_{1 \mathrm{a}}: \beta_{0}>0, H_{0}: \beta_{0} \leq 0 . \\
& H_{1 \mathrm{~b}}: \beta_{1}>0, H_{0}: \beta_{1} \leq 0 .
\end{aligned}
$$

Second, I test whether warm glow and pure altruism vary by demographic group. Hypotheses 2a and 2b: Giving motivated by warm glow and pure altruism respectively vary by gender.

$$
\begin{aligned}
& H_{2 a}: \beta_{0} \mid \text { male } \neq \beta_{0} \mid \text { female }, H_{0}: \beta_{0} \mid \text { male }=\beta_{0} \mid \text { female } . \\
& H_{2 \mathrm{~b}}: \beta_{1} \mid \text { male } \neq \beta_{1} \mid \text { female }, H_{0}: \beta_{1} \mid \text { male }=\beta_{1} \mid \text { female } .
\end{aligned}
$$

Hypotheses 3a and 3b: Giving motivated by warm glow and pure altruism respectively vary by age. 


$$
\begin{aligned}
& H_{3 \mathrm{a}}: \beta_{0} \text { |age } \neq \beta_{0}, H_{0}: \beta_{0} \text { |age }=\beta_{0} . \\
& H_{3 \mathrm{~b}}: \beta_{1} \text { |age } \neq \beta_{1}, H_{0}: \beta_{1} \text { |age }=\beta_{1} .
\end{aligned}
$$

Hypothesis 4a and 4b: Giving motivated by warm glow and pure altruism respectively vary by income.

$$
\begin{aligned}
& H_{4 a}: \beta_{0} \mid \text { income } \neq \beta_{0}, H_{0}: \beta_{0} \mid \text { income }=\beta_{0} . \\
& H_{4 b}: \beta_{1} \mid \text { income } \neq \beta_{1}, H_{0}: \beta_{1} \mid \text { income }=\beta_{1} .
\end{aligned}
$$

\section{Experiment Design}

\subsection{Treatments}

The experiment is structured as a dictator game with a variable multiplication factor which increases or decreases the amount received by a randomly paired passive counterpart for a given amount sent. Dictators receive an initial endowment of $\$ 1.00$ and are instructed that they may send any portion of the endowment to a randomly selected subject in another group. They are then presented with a list of multiplication factors $\mu \in\{0.2,0.4,0.6,0.8,1,2,3,4,5\}$ and are asked to choose the amount they would send for each value of $\mu$. They are told that after making their choices, one choice will be randomly selected to be exectuted. Multiplication factors are presented on a single page, with the order randomly set as high to low or low to high. Instructions as seen by subjects are included in appendix $\mathrm{A}^{2}$.

Prior to starting the experiment, prospective subjects are asked to answer this question: "You have a basket containing five apples. You eat one apple and sell two apples. How many apples are now in your basket?" This question serves to prevent automated scripts from entering the experiment, similar in function to $\mathrm{CAPTCHA}^{3}$ codes seen on websites where automated spam is a concern. Subjects are not allowed to enter the experiment without entering the correct answer. Next, subjects are shown instructions followed by a pair of questions to test understanding ${ }^{4}$. After answering the understanding question, subjects are shown the correct answer with an explanation of why it is correct. Unlike the automated script filter question,

\footnotetext{
${ }^{2}$ Appendices and supplemental materials are available at https://robertmayo.wordpress.com/research-2/

${ }^{3}$ Information and examples of the use of CAPTCHA systems is available at https://www.google.com/recaptcha/intro/index.html.

${ }^{4} 85 \%$ and $65 \%$ of subjects answered the respective understanding questions correctly prior to being shown the correct answers and explanations.
} 
subjects are allowed to proceed to the main body of the experiment regardless of whether they answered the understanding question correctly or incorrectly. After entering the amounts they would send to a randomly paired subject in a second group for each value of $\mu$, subjects are presented with an exit questionnaire which collects basic demographic data. They are also invited to add an unstructured message about any technical difficulty encountered in the experiment or anything else they wish to share. They are then shown their payoff and the experiment concludes. For reasons discussed in the next section, subjects in the recipient group are recruited in a separate asynchronous session.

\subsection{Infrastructure}

The design described in the previous section was conducted over the internet using two main technologies.

\subsection{1. oTree}

oTree (Chen, Schonger, and Wickens 2016) is a software platform that allows multiplayer decision experiments to be conducted over the internet. oTree experiments are coded in Python and use the Django web framework. ${ }^{5}$ Experiment development is done locally then uploaded to a remote web server along with the oTree supporting files. I used the commercial web hosting company Heroku ${ }^{6}$ for hosting services. After server deployment, experiments are run through a web-based administrative interface. Since the experiment runs on a remote web server, subjects can enter the experiment from any location. Participation is not limited to any particular operating system or device form factor. All that is required is a browser with internet access.

Python, the language used to code oTree experiments, is a popular general purpose computer language which is well suited and commonly used for web development. It has the added advantage of being user friendly and relatively easy to learn. oTree uses Python on top of the Django web framework ${ }^{7}$. However, Django is designed for professional level web development and requires a commensurate level of technical knowledge to navigate. Also,

\footnotetext{
5 Technical details of Django can be found at https://www.djangoproject.com/.

${ }^{6}$ Technical details of Heroku can be found at https://www.heroku.com/.

${ }^{7} \mathrm{~A}$ web framework is a collection of functions that automate common development tasks.
} 
prospective users of oTree should be aware that as of this writing it is still under development and so should be considered a work in progress.

\subsubsection{Mechanical Turk}

Subject recruitment and payment was done through the Mechanical Turk microemployment website. Mechanical Turk is an Amazon service which allows workers to perform small tasks for modest compensation. In Mechanical Turk terminology, a task is a Human Intelligence Task or HIT. Employers, called Requesters, register with the service and deposit funds to pay workers to perform HITs. Typical HITs are very easy for a human to do but very difficult to automate, such as identifying objectionable content in user generated web posts or classifying photographs by subject. Thus, the Mechanical Turk marketing phrase "Artificial, artificial intelligence”.

The experiment is posted to Mechanical Turk as a HIT using IRB approved advertisement language. Workers who wish to participate accept the HIT and are transferred to the experiment website. After completing the experiment, subjects are returned to the Mechanical Turk website and are free to proceed to their next chosen HIT. Since there is no face to face interaction between requester and worker, automated scripts deployed to accept HITs and input random responses to generate payments are a possibility. It is for this reason that the script filtering question discussed in the previous section is a wise precaution. It has the added advantage of screening out persons who enter random responses, essentially mashing the keyboard in an attempt to receive payment with minimum time and effort.

Interaction between dictators and recipients are done asynchronously. All dictators make their choices of how much to send, then at a later time a second group of Mechanical Turk workers is recruited as passive recipients. Participants in the dictator group are ineligible to participate in the recipient group. Since the information flow is only from dictator to recipient, the small time delay this method imposes between money sent and money received should not bias dictator choices. This method was chosen due to a peculiarity of Mechanical Turk. After posting a HIT, workers will see and accept the HIT with a variable delay of between a few seconds and a few minutes, depending on the number of workers searching for HITs at the time. If after accepting the HIT workers were paired with another worker, then whichever worker was the first of the pair to arrive would be unable to proceed into the experiment until the next worker 
accepted the HIT and completed the pair. If the rate of arrival exceeds a few seconds, workers with higher levels of impatience may preferentially return the HIT or otherwise exit believing that the wait may be excessive or that the HIT is malfunctioning. This could initiate a cascade of workers being unable to be paired in a reasonable amount of time causing the experiment to fail. Experiments have been run successfully on Mechanical Turk using real-time interaction between group members (Mayo, McCabe, and Kreuger 2016), but this issue must be taken into consideration in experiment design.

\subsection{Sample and randomization}

No personally identifying information is passed from worker to requester other than a unique Mechanical Turk worker identification number. Amazon securely stores workers' personal information including financial information required to process payments. This simplifies experimenter record keeping and ensures subject privacy, but also raises the possibility of workers attempting to participate in the experiment repeatedly. Through the oTree API, a worker qualification can be set. This tells Mechanical Turk that the requester only wants the HIT to be accessible by workers who meet certain criteria. The two criteria used in this experiment were that the worker must reside in the United States and not have previously participated in the experiment. Amazon verifies workers' country of residence by requiring payments to workers claiming United States residence be deposited electronically to a United States bank account. The association between bank account and worker ID number also restricts the ability of one worker holding more than one Mechanical Turk worker account. Amazon also uses proprietary methods of fraud control not publicly disclosed.

Workers are not able to access any information about the content of the experiment prior to accepting the HIT assignment, other than IRB risk disclosure and a description of the HIT as an academic experiment in decision making. Therefore there is little reason to suspect significant bias in results caused by self selection into the experiment. The on-line environment does, however, raise methodological questions about the external validity of experiments conducted without substantial control of subjects' environments (Horton, Rand, and Zeckhauser 2011). In addition, stakes in on-line experiments using Mechanical Turk can be an order of magnitude lower than the same experiment would require if conducted in an in-person laboratory setting. Amir, Rand, and Gal (2012) studied both of these issues by replicating a series of classic 
economic experiments in an on-line environment and found no significant difference between the behavior of on-line and in-person subjects. Fortuitously, the stakes studied in the on-line treatments were the same as used in this experiment, $\$ 1.00$.

Another issue of potential concern is the demographic composition of the subject pool and how it may differ from the demographics of subjects available for in-person experimentation in ways that might call into question the generalizability of results. Berinsky, Huber, and Lenz (2012) examined the demographics of Mechanical Turk workers who participated in experiments and found the distribution to be closer to that of the United States population than is found in subjects reported in a group of in-person studies published in major political science journals.

\section{Results}

\subsection{Summary statistics}

The experiment was run over four consecutive weekdays. 146 Mechanical Turk workers accepted the HIT and $112^{8}$ completed the experiment giving a dropout rate of $23 \%$. This dropout rate is quite high when compared to the typical in-person laboratory experiment, but is actually good for on-line experiments. Dropout rates of over $50 \%$ are not uncommon in on-line experiments (Dandurand, Shultz, and Onishi 2008). This is understandable given the lack of social sanction for exiting an experiment prematurely by simply closing a browser window.

The sample has a mean age of 34 and has reasonable gender balance at $59 \%$ male and $41 \%$ female. Mean income is $\$ 53,600$ per year. Summary statistics are shown in table 1 . Mean amounts sent by multiplier and demographic group are shown in table 3. Age and income demographic groups are defined as above or below their mean values.

Table 1. Summary statistics.

\begin{tabular}{lrrrr}
\hline & Mean & St. Dev. & Min & Max \\
\hline Age & 34.14 & 9.99 & 18.00 & 59.00 \\
Income (in thousands) & $\$ 53.60$ & $\$ 45.96$ & $\$ 0.00$ & $\$ 250.00$ \\
Female & 0.41 & & & \\
$\mathrm{~N}$ & 112 & & & \\
\hline
\end{tabular}

${ }^{8}$ Although online experiments are typically less costly than laboratory experiments, sample size was nonetheless constrained by the research budget. 
Table 2. Pairwise demographic correlations.

\begin{tabular}{llll}
\hline & Age & Gender $^{\dagger}$ & Income \\
\hline Age & 1.000 & & \\
Gender & 0.2984 & 1.000 & \\
Income & 0.0823 & -0.0101 & 1.000 \\
\hline 'Female $=$ & 1, Male $=0$ & & \\
\hline
\end{tabular}

Table 3. Mean amounts sent by multiplier and demographic group.

\begin{tabular}{|c|c|c|c|c|c|c|c|c|c|c|}
\hline \multirow[b]{2}{*}{ Group } & \multirow[b]{2}{*}{$\mathrm{N}$} & \multicolumn{9}{|c|}{ Multiplier $\mu$} \\
\hline & & 0.2 & 0.4 & 0.6 & 0.8 & 1 & 2 & 3 & 4 & 5 \\
\hline All & 112 & $\$ 0.24$ & $\$ 0.23$ & $\$ 0.25$ & $\$ 0.26$ & $\$ 0.31$ & $\$ 0.31$ & $\$ 0.31$ & $\$ 0.31$ & $\$ 0.32$ \\
\hline Male & 66 & $\$ 0.20$ & $\$ 0.20$ & $\$ 0.23$ & $\$ 0.25$ & $\$ 0.31$ & $\$ 0.32$ & $\$ 0.34$ & $\$ 0.34$ & $\$ 0.34$ \\
\hline Female & 46 & $\$ 0.28$ & $\$ 0.27$ & $\$ 0.29$ & $\$ 0.27$ & $\$ 0.33$ & $\$ 0.30$ & $\$ 0.28$ & $\$ 0.26$ & $\$ 0.29$ \\
\hline Age $<34$ & 65 & $\$ 0.20$ & $\$ 0.21$ & $\$ 0.25$ & $\$ 0.25$ & $\$ 0.30$ & $\$ 0.32$ & $\$ 0.31$ & $\$ 0.31$ & $\$ 0.33$ \\
\hline Age $\geq 34$ & 47 & $\$ 0.28$ & $\$ 0.26$ & $\$ 0.26$ & $\$ 0.28$ & $\$ 0.34$ & $\$ 0.31$ & $\$ 0.32$ & $\$ 0.30$ & $\$ 0.32$ \\
\hline Income $<\$ 53.6 \mathrm{k}$ & 70 & $\$ 0.21$ & $\$ 0.21$ & $\$ 0.24$ & $\$ 0.25$ & $\$ 0.31$ & $\$ 0.31$ & $\$ 0.31$ & $\$ 0.32$ & $\$ 0.33$ \\
\hline Income $\geq \$ 53.6 \mathrm{k}$ & 42 & $\$ 0.28$ & $\$ 0.26$ & $\$ 0.28$ & $\$ 0.28$ & $\$ 0.33$ & $\$ 0.32$ & $\$ 0.32$ & $\$ 0.30$ & $\$ 0.32$ \\
\hline
\end{tabular}

\subsection{Motivation for giving}

Quantile regression of amounts sent on multiplication factor for each dictator in the full sample produced within subject estimates of the coefficient on the variable $\mu$ representing the effect of changing the price of benefit to the recipient in the dictator's choice of amount to send, and a constant term representing the choice of amount to send that was not a non-constant function of the price. These values estimate the amounts sent motivated by warm glow (constant term) and pure altruism (coefficient on $\mu$ ). A one-sample t-test showed the regression constant terms to be greater than zero, $($ Mean $=0.230, S E=0.027, t(111)=8.373, p=0.000)$. A second one-sample t-test showed the coefficients on $\mu$ to also be greater than zero, $($ Mean $=0.020, S E=$ $0.007, t(111)=2.764, p=0.007)$. Detailed test results are shown in appendix B. Since both the coefficient on the multiplier and the constant terms are significantly greater than zero at the 0.01 confidence level, the nulls of hypotheses $1 \mathrm{a}$ and $1 \mathrm{~b}$ can be safely rejected. This confirms the existence of both pure altruism and warm glow in the full sample. The fraction of total giving 
motivated by pure altruism (Andreoni's $\alpha$ ) in the full sample was slightly over one-third, (Mean $=0.379, S D=0.730$ ). The distribution of $\alpha$ is shown in figure 1 .

Figure 1.

Kernel density plot of $\alpha$ for full sample.

Kernel is Gaussian, bandwidth $=0.2$.

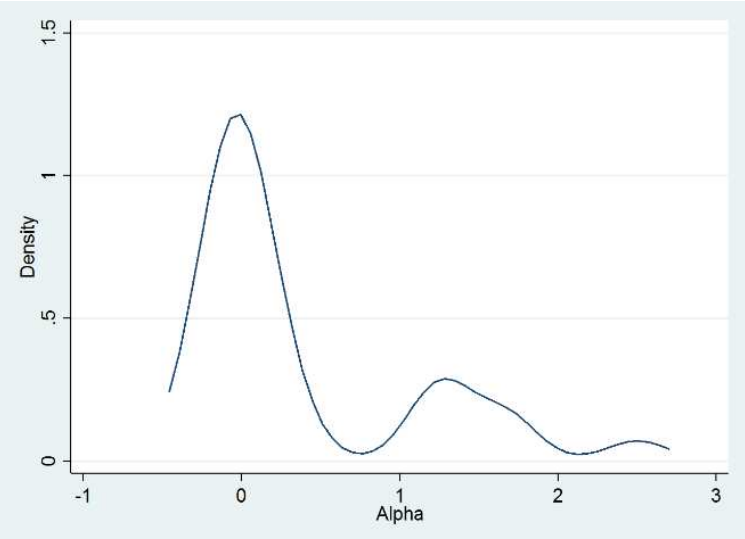

\subsection{Motivation by age}

Pearson's $\mathrm{r}$ showed giving motivated by warm glow was slightly declining with the age of the dictator, but the effect was not statistically significant. $(r=-0.0047, n=112, p=0.961)$. Similarly, giving motivated by pure altruism was slightly increasing with the age of the dictator and was also not statistically significant. $(r=0.0012, n=112, p=0.990)$. Since age is not a significant predictor of either amounts sent due to warm glow or amounts sent due to pure altruism, the nulls of hypotheses $3 \mathrm{a}$ and $3 \mathrm{~b}$ are not rejected. A test of $\alpha$ also showed no significant correlation with age, $(r=0.001, n=112, p=0.995)$. Warm glow, pure altruism, and $\alpha$ as functions of age are shown in figures 2,3 , and 4 respectively. 
Figure 2.

Warm glow as a function of age.

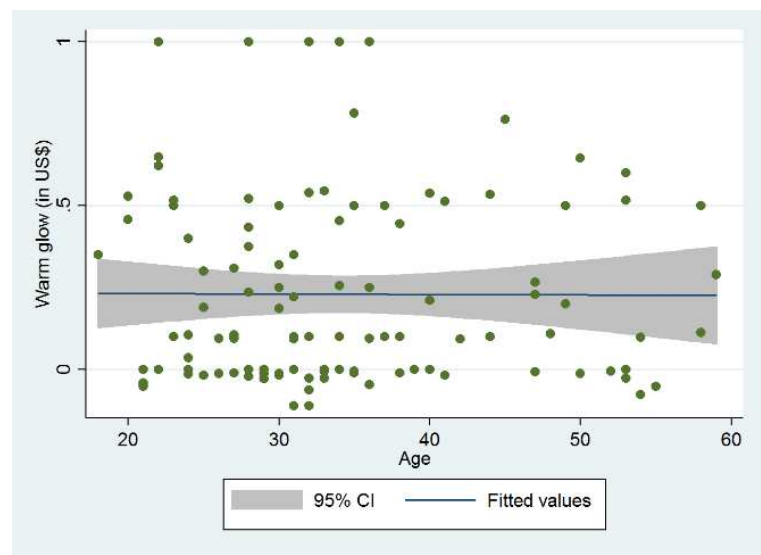

Figure 3.

Pure altruism as a function of age.

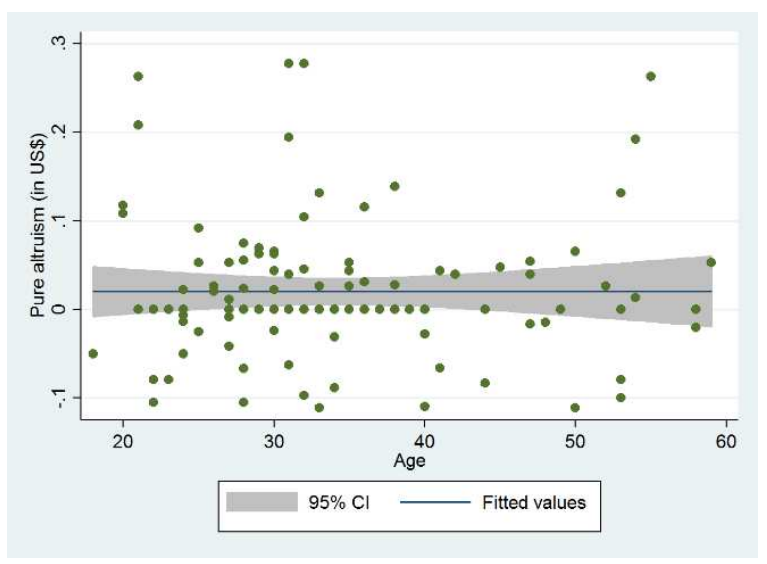

Figure 4.

$\alpha$ as a function of age.

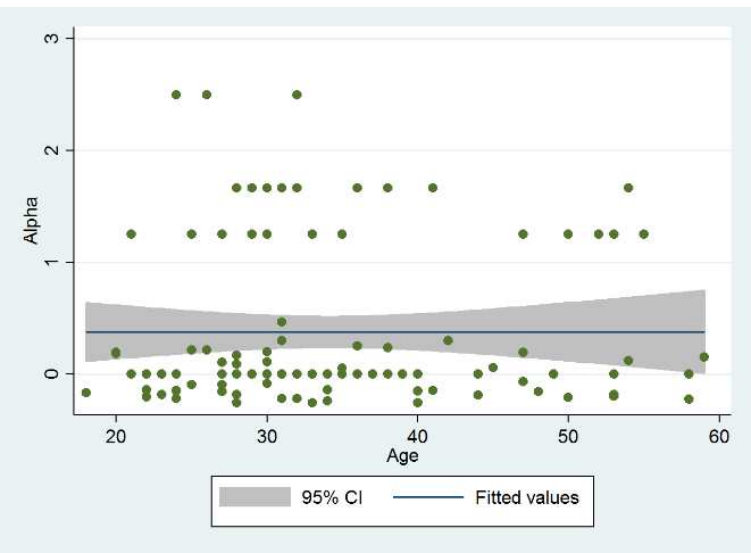




\subsection{Motivation by income}

Pearson's $r$ showed a small positive correlation between warm glow and income that was not statistically significant, $(r=0.037, n=112, p=0.697)$. Pure altruism was also positively correlated with income and not statistically significant, $(r=0.017, n=112, p=0.857)$. Finally, $\alpha$ was slightly, but not significantly, increasing in income, $(r=0.034, n=112, p=0.719)$. Warm glow, pure altruism, and $\alpha$ as functions of income are shown in figures 5, 6, and 7 .

Figure 5.

Warm glow as a function of income.

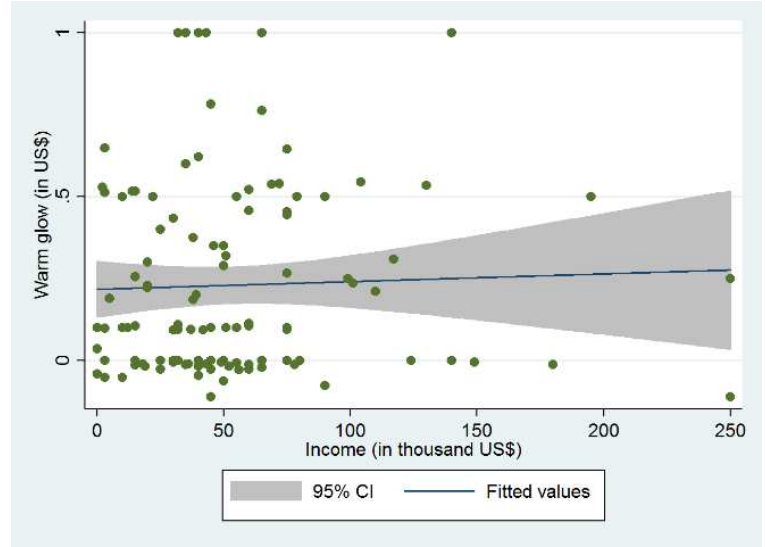

Figure 6 .

Pure altruism as a function of income.

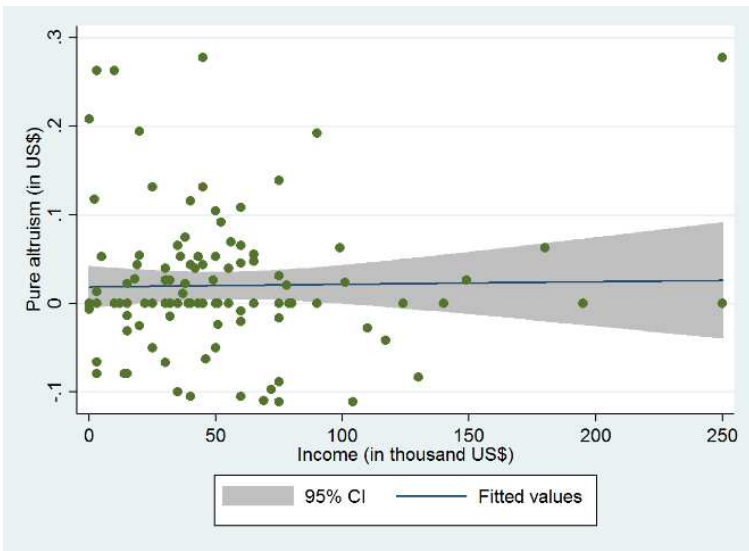

Figure 7.

$\alpha$ as a function of income.

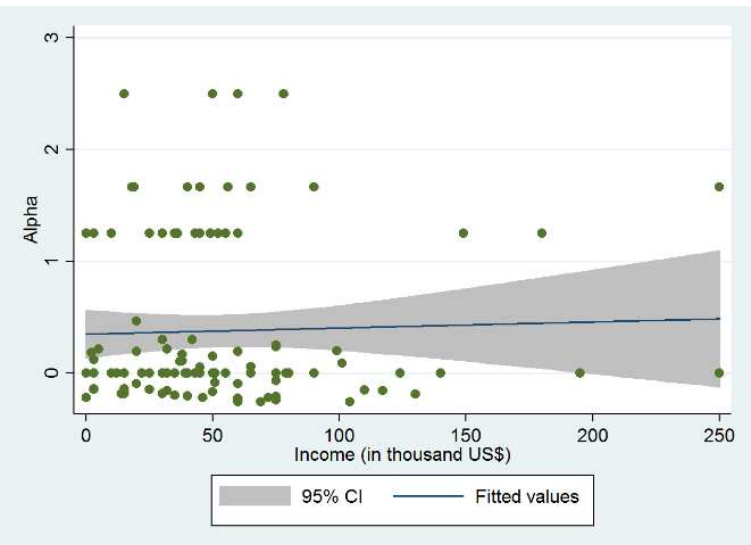




\subsection{Motivation by gender}

Women in the sample sent more due to warm glow $($ mean $=\$ 0.27)$ than did men $($ mean $=\$ 0.20)$. However, a two-sample t-test did not find this difference to be significant, $(t=-1.280$, $n($ male $)=66, n($ female $)=46, p=0.204)$. By contrast, women sent essentially nothing due to pure altruism $($ mean $=\$ 0.001)$ compared to men $($ mean $=\$ 0.034)$. This difference was statistically significant, $(t=2.332, n$ (male $)=66, n($ female $)=46, p=0.022)$. $\alpha$ was higher for men (0.469) than for women $(0.250)$, but this difference was of borderline significance, $(t=$ 1.613, $n($ male $)=66, n($ female $)=46, p=0.110)$. Warm glow, pure altruism, and $\alpha$ by gender are shown in figures 8,9 , and 10 . Figure 11 shows the amounts sent as a function of the multiplier $\mu$ for men and for women.

Figure 8.

Warm glow by gender.

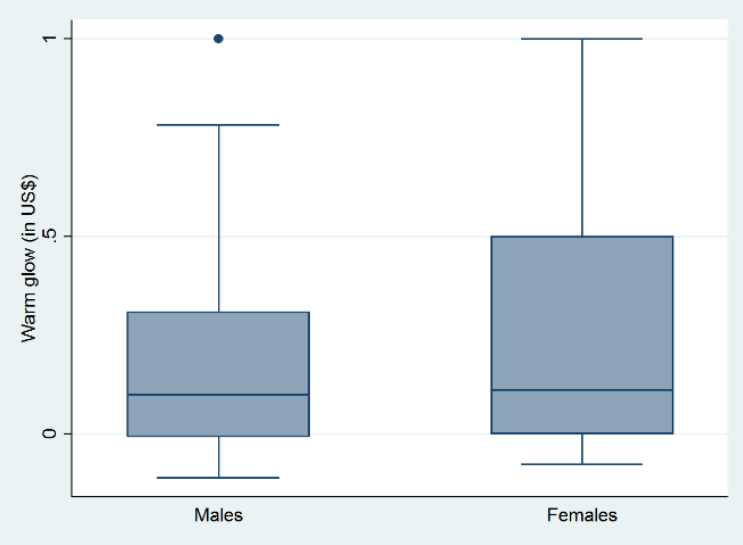

Figure 10.

$\alpha$ by gender.

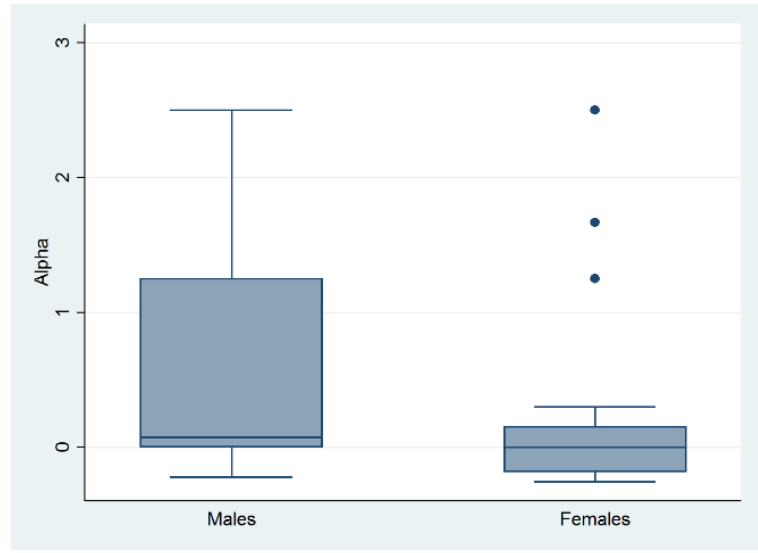

Figure 9.

Pure altruism by gender.

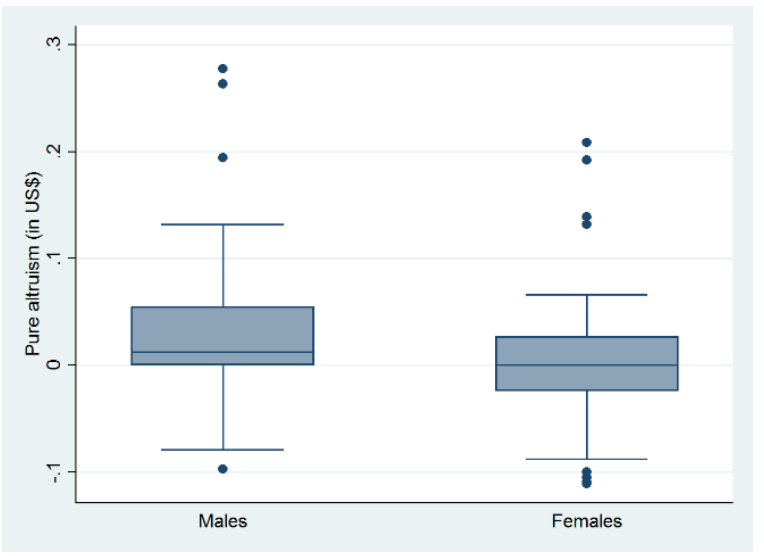

Figure 11.

Amounts sent as a function of the multiplier $\mu$ for men and for women. 


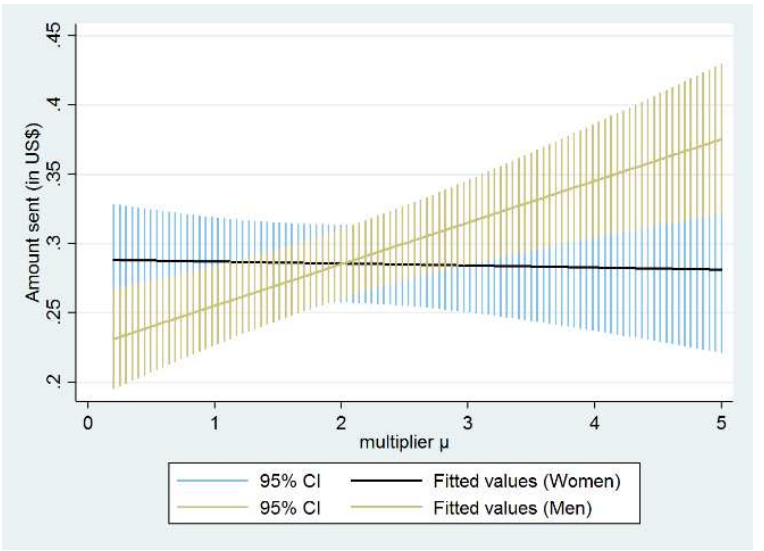

\section{Discussion}

The results reported in the previous section confirm that both warm glow and pure altruism are motivating factors in subjects' decisions to give to an an anonymous, paired subject. These results confirms the existence of both warm glow and pure altruism that has been found in several prior studies. Palfrey and Prisbrey (1997) found evidence of warm glow, but not of pure altruism as a motivation for giving in a public goods game. By contrast, Goeree, Holt, and Laury (2002) found evidence of pure altruism, but not of warm glow as motivating factors in subjects' behavior. And Crumpler and Grossman (2008) found strong evidence of warm glow as a mativator, but did not test for the existence of pure altruism. Beyond establishing the existence of both effects, by multiplying mean amounts sent by mean $\alpha$ for each gender, I find that warm glow is responsible for the majority of giving (61\%) with pure altruism composing a minority share (39\%). This contradicts the findings of Lilley and Slonim (2014) which quantified the relative influence of the two motivators and found that that larger share of giving was due to pure altruism (79\%) with only $21 \%$ resulting from warm glow. By contrast, Eckel and Grossman (1996) found that men were largely insensitive to changes in the price of punishment.

Examining results by demographic group shows no significant relationship between motivation and either the age or income of the dictator. Although, both giving due to pure altruism and warm glow were slightly increasing in income which is unsurprising since total giving also increased slightly with income. Other than showing that both motivators exist, the major result is the difference in motivation by gender. Although males and females were equally generous when the multiplier effect was absent $(\mu=1.0)$ with males giving $\$ 0.31$ and females 
giving \$0.33, their behavior diverged significantly at other multiplier levels. At the lowest multiplier value where only $20 \%$ of the amount sent reached the recipient, females still gave $\$ 0.28$, while males by contrast reduced their giving to $\$ 0.20$. At the highest multiplier value, where the recipient received $500 \%$ of the amount sent, females sent almost exactly the same amoun $\$ 0.29$. Males responded to the highest multiplier by increasing their amounts sent to $\$ 0.34$. This implies that females are motivated almost entirely by warm glow, while men are significantly motivated by both warm glow and pure altruism. This gender difference is broadly consistent with the findings of Tonin and Vlassopoulos (2010) which found warm glow to be a significant factor for women but not for men, although for neither gender do they find evidence of pure altruism. Andreoni and Vesterlund (2001) also find that males are much more responsive to the price of benefit to the recipient, while females tend to send the same amount regardless of price. By contrast,

\subsection{Alternative explanations and possible objections}

The decomposition of motives into warm glow and pure altruism depends on the assumption that warm glow giving is unrelated to the amount received by the passive subject. It is possible that this is not true. If, for example, a dictator derived utility from the amount received by the passive player but had no concern for the welfare of that player, then the dictator could be highly responsive to price and simultaneously motivated only by warm glow. If so, then values generated by this model for giving motivated by warm glow would be a lower bound and values for $\alpha$ would be an upper bound. Although this is possible, it would require a substantial revision of the impure altruism model that is beyond the scope of this paper. It is also possible that a person giving a fixed percentage of their endowment regardless of the amount received by an anonymous counterpart may be motivated by concern for the recipient, but effecting that goal through a tithing rule, as was seen in Eckel and Grossman (2004).

The major results in terms of demographic differences could be artifacts of some form of self selection by workers prior to accepting the HIT or afterwards within the $20 \%$ who began the study but did not complete it. Selection prior to accepting the HIT is unlikely because the advertisement language was deliberatly vague about the nature of the experiment, but absence of evidence is not evidence of absence so the possibility cannot be ruled out. 


\section{Conclusion}

This paper reports the results of an on-line experiment designed to disaggregate giving in a dictator game into the portion motivated by pure altruism and the portion motivated by warm glow, as described in Andreoni (1989). By manipulating the price of benefit to the recipient while holding the price of the act of giving constant, I am able to estimate values for a coefficient of altruism $\alpha$ (Ibid. p1452). I find significant evidence of both pure altruism and warm glow as motivations for giving. In addition, I repeat the statistical estimation on sub-sets of the sample based on demographic criteria as reported in an exit survey. The fraction of amounts sent to an anonymous recipient that are motivated by warm glow is uncorrelated with the age of the dictator and is increasing in dictator's income, but the relationship is not statistically significant. An analysis by gender shows that male subjects responded strongly to price suggesting they are mostly motivated by pure altruism, while female subjects were almost completely insensitive to price suggesting a large fraction of giving motivated by warm glow and little motivated by pure altruism.

\section{References}

Amir, Ofra, David G Rand, and Ya'akov Kobi Gal. 2012. "Economic Games on the Internet: The Effect of \$1 Stakes.” PLoS ONE 7 (2): 1-4. doi:10.1371/journal.pone.0031461.

Andreoni, James. 1989. "Giving with Impure Altruism: Applications to Charity and Ricardian Equivalence.” Journal of Political Economy 97 (6): 1447. doi:10.1086/261662.

Andreoni, James, and Lise Vesterlund. 2001. "Which Is the Fair Sex? Gender Differences in Altruism.” The Quarterly Journal of Economics 116 (1): 293-312. doi:10.1162/003355301556419.

Arabmazar, Abbas, and Peter Schmidt. 1982. "An Investigation of the Robustness of the Tobit Estimator to Non-Normality." Econometrica 50 (4): 1055-63. http://www.jstor.org/stable/1912776.

Berinsky, Adam J., Gregory A. Huber, and Gabriel S. Lenz. 2012. "Evaluating Online Labor Markets for Experimental Research: Amazon.com's Mechanical Turk.” Political Analysis 
20 (3): 351-68. doi:10.1093/pan/mpr057.

Brown, Alexander L, Jonathan Meer, and J Forrest Williams. 2013. "Why Do People Volunteer? An Experimental Analysis of Preferences for Time Donations." NBER.

Chen, Daniel L., Martin Schonger, and Chris Wickens. 2016. "oTree-An Open-Source Platform for Laboratory, Online, and Field Experiments." Journal of Behavioral and Experimental Finance 9. Elsevier B.V.: 88-97. doi:10.1016/j.jbef.2015.12.001.

Crumpler, Heidi, and Philip J. Grossman. 2008. "An Experimental Test of Warm Glow Giving." Journal of Public Economics 92 (5-6): 1011-21. doi:10.1016/j.jpubeco.2007.12.014.

Dandurand, Frédéric, Thomas R. Shultz, and Kristine H. Onishi. 2008. "Comparing Online and Lab Methods in a Problem-Solving Experiment.” Behavior Research Methods 40 (2): 428 34. doi:10.3758/BRM.40.2.428.

Doebeli, Michael, and Christoph Hauert. 2005. "Models of Cooperation Based on the Prisoner's Dilemma and the Snowdrift Game." Ecology Letters 8 (7): 748-66. doi:10.1111/j.14610248.2005.00773.x.

Eckel, Catherine C., and Philip J. Grossman. 1996. "The Relative Price of Fairness : Gender Differences in a Punishment Game Punishment Game '." Journal of Economic Behavior \& Organisation 30: 143-58.

Eckel, Catherine C, and Philip J Grossman. 2003. "Rebate versus Matching : Does How We Subsidize Charitable Contributions Matter ?" Journal of Public Economics 87: 681-701. doi:10.1016/S0047-2727(01)00094-9.

_ 2004. "Giving to Secular Causes by the Religious and Nonreligious : An Experimental Test of the Responsiveness of Giving to Subsidies." Nonprofit and Voluntary Sector Quarterly 33 (2): 271-89. doi:10.1177/0899764004263423.

Engel, Christoph. 2011. "Dictator Games: A Meta Study.” Experimental Economics 14 (4): $583-$ 610. doi:10.1007/s10683-011-9283-7.

Gangadharan, Lata, Philip J Grossman, and Kristy Jones. 2014. "Deconstructing Giving : Donor Types and How They Give.” Science of Philanthropy Initiative. 
Goeree, Jacob K., Charles A. Holt, and Susan K. Laury. 2002. "Private Costs and Public Benefits: Unraveling the Effects of Altruism and Noisy Behavior." Journal of Public Economics 83 (2): 255-76. doi:10.1016/S0047-2727(00)00160-2.

Greene, William H. 2008. Econometric Analysis. 7th ed. Upper Saddle River, N.J.: Prentice Hall. Horton, John J., David G. Rand, and Richard J. Zeckhauser. 2011. "The Online Laboratory: Conducting Experiments in a Real Labor Market.” Experimental Economics 14 (3): 399 425. doi:10.1007/s10683-011-9273-9.

Karlan, By Dean, and John A List. 2005. "Does Price Matter in Charitable Giving ? Evidence from a Large-Scale Natural Field Experiment,” no. 2002.

Konow, James. 2010. "Mixed Feelings : Theories of and Evidence on Giving.” Journal of Public Economics 94 (3-4). Elsevier B.V.: 279-97. doi:10.1016/j.jpubeco.2009.11.008.

Lilley, Andrew, and Robert Slonim. 2014. "The Price of Warm Glow." Journal of Public Economics 114 (7445): 58-74. doi:10.1016/j.jpubeco.2013.12.004.

List, John A, Sally Sadoff, and Mathis Wagner. 2011. "So You Want to Run an Experiment, Now What? Some Simple Rules of Thumb for Optimal Experimental Design," 439-57. doi:10.1007/s10683-011-9275-7.

Luccasen, Andrew, and Philip J Grossman. 2017. "Warm-Glow Giving : Earned Money and the Option to Take.” Economic Inquiry 55 (2): 996-1006. doi:10.1111/ecin.12417.

Mayo, Robert L, Kevin McCabe, and Frank Kreuger. 2016. "Replicating the Triadic Trust Experiment on Mechanical Turk.” (Under Review).

Palfrey, Thomas, and Jeffrey Prisbrey. 1997. "Anomalous Behavior in Public Goods Experiments : How Much and Why ?" The American Economic Review 87 (5): 829-46. http://www.jstor.org/stable/2951327.

Portnoy, Stephen. 2016. “Censored Regression Quantiles” 98 (464): 1001-12. doi:10.1198/016214503000000954.

Ribar, David C, and Mark O Wilhelm. 2002. "Altruistic and Joy-of-Giving Motivations in Charitable Behavior.” Journal of Political Economy 110 (2): 425-57. 
Rigobon, Roberto, and Thomas M. Stoker. 2009. "Bias From Censored Regressors." Journal of Business \& Economic Statistics 27 (3): 340-53. doi:10.1198/jbes.2009.06119.

Smith, Adam. 1904. An Inquiry into the Nature and Causes of the Wealth of Nations. Edited by Edwin Cannan. London: Methuen \& Co., Ltd.

— 1982. The Theory of Moral Sentiments. Edited by D. D. Raphael and A. L. Macfie. London: Oxford University Press.

Tonin, Mirco, and Michael Vlassopoulos. 2010. "Disentangling the Sources of pro-Socially Motivated Effort: A Field Experiment." Journal of Public Economics 94 (11-12). Elsevier B.V.: 1086-92. doi:10.1016/j.jpubeco.2010.08.011.

—. 2014. "An Experimental Investigation of Intrinsic Motivations for Giving," 47-67. doi:10.1007/s11238-013-9360-9.

Zelmer, Jennifer. 2003. "Linear Public Goods Experiments: A Meta-Analysis.” Experimental Economics 6 (3): 299-310. doi:10.1023/A:1026277420119. 


\section{Appendix A. Experiment Interface}

\section{Subject screen 1}

Test Question

Time left to complete this page: $\odot 0: 55$

To prove that you are a human, please answer this question:

You have a basket containing five apples. You eat one apple and sell two apples.

How many apples are now in your basket?

Please enter your answer here:

\section{Subject screen 2}

Instructions

You start with $\$ 1.00$. You will be asked to choose how much of this $\$ 1.00$ you wish to send to a randomly selected person in another group. Any amount you send will be multiplied by the experimenter by a multiplication factor. The table below shows examples of how this works.

\begin{tabular}{|c|c|c|}
\hline If you send & And the mutiplication factor is & The other person would receive \\
\hline$\$ 1.00$ & $10 \%$ & $\$ 0.10$ \\
\hline$\$ 1.00$ & $50 \%$ & $\$ 0.50$ \\
\hline$\$ 1.00$ & $100 \%$ & $\$ 1.00$ \\
\hline$\$ 1.00$ & $150 \%$ & $\$ 1.50$ \\
\hline$\$ 1.00$ & $300 \%$ & $\$ 3.00$ \\
\hline
\end{tabular}

You will be asked to choose the amount you would send for a variety of different multiplications factors. At the end of the experiment, ONE of your choices will be picked at random and paid.

For your convenience, these instructions will remain available to you on all subsequent screens of this study.

On the next page, you will have to answer one question to make sure you understand the instructions correctly. 


\section{Subject screen 3 \\ Understanding Test}

If you start with $\$ 1.00$, decide to send $\$ 0.20$ to the other person and the multiplication factor is $50 \%$ how much would you each have? How much would you have?

$\$ \quad 0.00$

How much would the other person have?

$\$ \quad 0.00$

Next

Subject screen 4

Answer

If you start with $\$ 1.00$, decide to send $\$ 0.20$ to the other person and the multiplication factor is $50 \%$ how much would you each have? You would have $\$ 1.00-\$ 0.20=\$ 0.80$, and the other person would have $0.5 \times \$ 0.20=\$ 0.10$.

Next 


\section{Subject screen 5}

\section{Amount to Send}

You have $\$ 1.00$. For each question below, decide how much you would send to the other person. One of your choices on this page will be randomly selected to be paid

If for any amount you send, the other person would receive $100 \%$ of that amount, how much of your $\$ 1.00$ would you choose to send? $\$$

If for any amount you send, the other person would receive $500 \%$ of that amount, how much of your $\$ 1.00$ would you choose to send? $\$$

If for any amount you send, the other person would receive $60 \%$ of that amount, how much of your $\$ 1.00$ would you choose to send? $\$$

If for any amount you send, the other person would receive $\mathbf{2 0} \%$ of that amount, how much of your $\$ 1.00$ would you choose to send? $\$$

If for any amount you send, the other person would receive $\mathbf{2 0 0} \%$ of that amount, how much of your $\$ 1.00$ would you choose to send? $\$$

If for any amount you send, the other person would receive $80 \%$ of that amount, how much of your $\$ 1.00$ would you choose to send? $\$$ $\$$

If for any amount you send, the other person would receive $300 \%$ of that amount, how much of your $\$ 1.00$ would you choose to send? $\$$

If for any amount you send, the other person would receive $400 \%$ of that amount, how much of your $\$ 1.00$ would you choose to send? $\$$ 


\section{Subject screen 6}

\section{Survey}

What is your age?

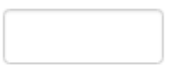

What is your gender?

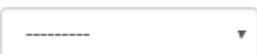

What is your racial or ethnic background?

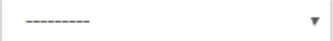

What is your marital status? :

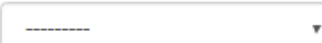

How would you best describe your current employment situation?

What is your household income (in thousands)?

0

How many people are in your household (including yourself)?

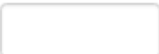

How many children are in your household?

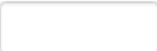

We would appreciate your comments about any technical problems, things that were unclear, ways this experiment could be improved, or any other feedback you wish to give. Thank you!

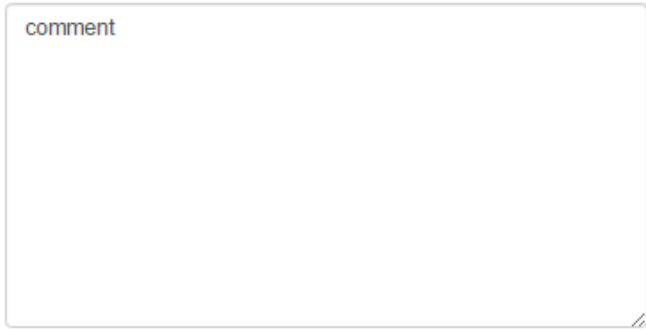




\section{Subject screen 7}

\section{Results}

You started with $\$ 1.00$. Your choice number 9 was randomly selected. You decided to send $\$ 1.00$ to the other person, leaving you with $\$ 0.00$. You will also receive $\$ 0.25$ for participation.

\section{Thank you!}

Next 


\section{Appendix B. Test Results Detail}

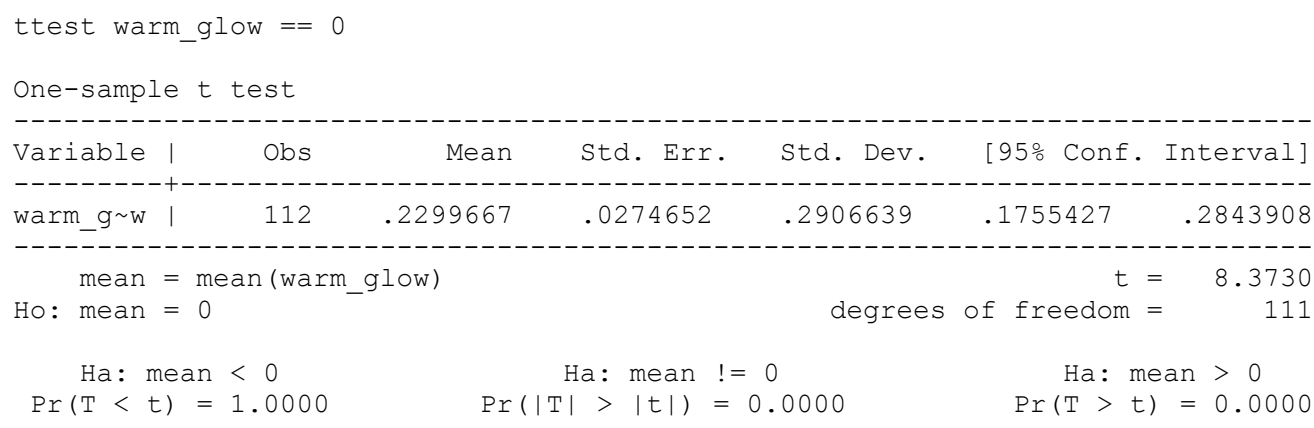

- ttest pure_altruism $==0$

One-sample $t$ test

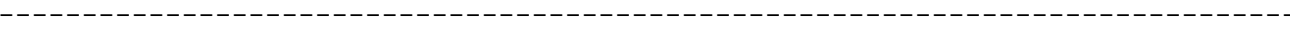

Variable | Obs Mean Std. Err. Std. Dev. [95\% Conf. Interval]

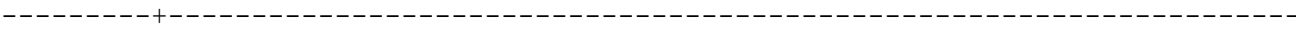

$\begin{array}{lllllll}\text { pure_a m | } & 112 & .0204058 & .0073824 & .0781276 & .0057772 & .0350345\end{array}$

pure a m ।

- summarize alpha

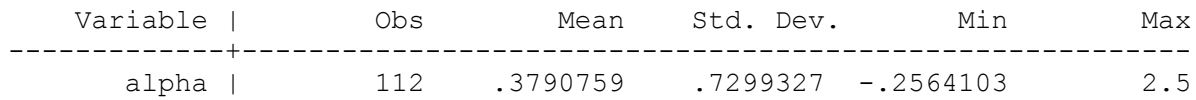

. pwcorr age warm_glow, sig star(.05) obs

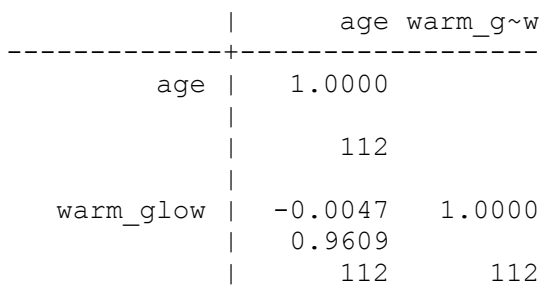

. pwcorr age pure_altruism, sig star(.05) obs

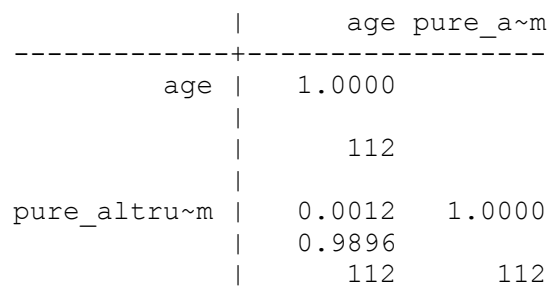

- pwcorr age alpha, sig star(.05) obs 


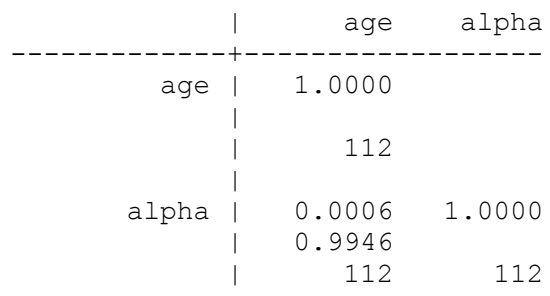

. pwcorr income warm_glow, sig star(.05) obs

\begin{tabular}{|c|c|c|c|}
\hline & । & income & arm_g w \\
\hline \multirow[t]{2}{*}{ income } & I & 1.0000 & \\
\hline & I & 112 & \\
\hline \multirow[t]{3}{*}{ warm_glow } & I & 0.0372 & 1.0000 \\
\hline & I & 0.6970 & \\
\hline & & 112 & 112 \\
\hline
\end{tabular}

. pwcorr income pure_altruism, sig star(.05) obs

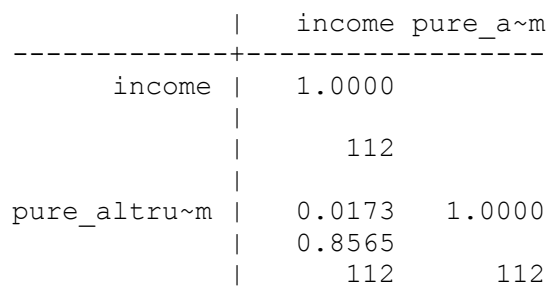

. pwcorr income alpha, sig star(.05) obs

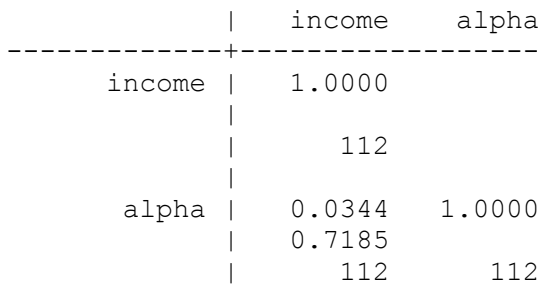

Two-sample $t$ test with unequal variances

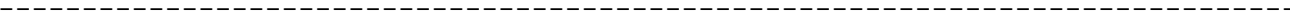

Group I Obs Mean Std. Err. Std. Dev. [95\% Conf. Interval]

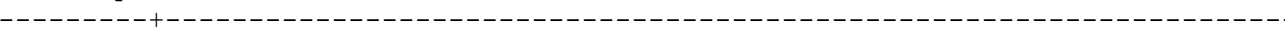

$\begin{array}{llllllll}0 & 1 & 66 & .2006072 & .0353998 & .2875897 & .1299089 & .2713056\end{array}$

$1 \mid \begin{array}{llllll}46 & .2720912 & .0432014 & .2930061 & .1850791 & .3591032\end{array}$

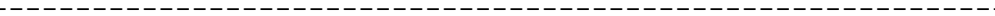

$\begin{array}{llllllll}\text { combined } & 112 & .2299667 & .0274652 & .2906639 & .1755427 & .2843908\end{array}$

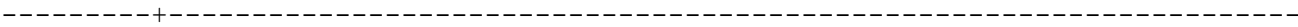

diff | - -.0714839 $\quad .0558526 \quad-.1823532 \quad .0393853$

-

mean $(1)$

Ho: diff $=0$

Satterthwaite's degrees of freedom $=95.8126$

Ha: diff $<0$

Ha: diff $!=0$

Ha: diff $>0$

$\operatorname{Pr}(T<t)=0.1018$

$\operatorname{Pr}(|\mathrm{T}|>|t|)=0.2037$

$\operatorname{Pr}(T>t)=0.8982$ 
- ttest pure altruism, by (female) unequal

Two-sample $t$ test with unequal variances

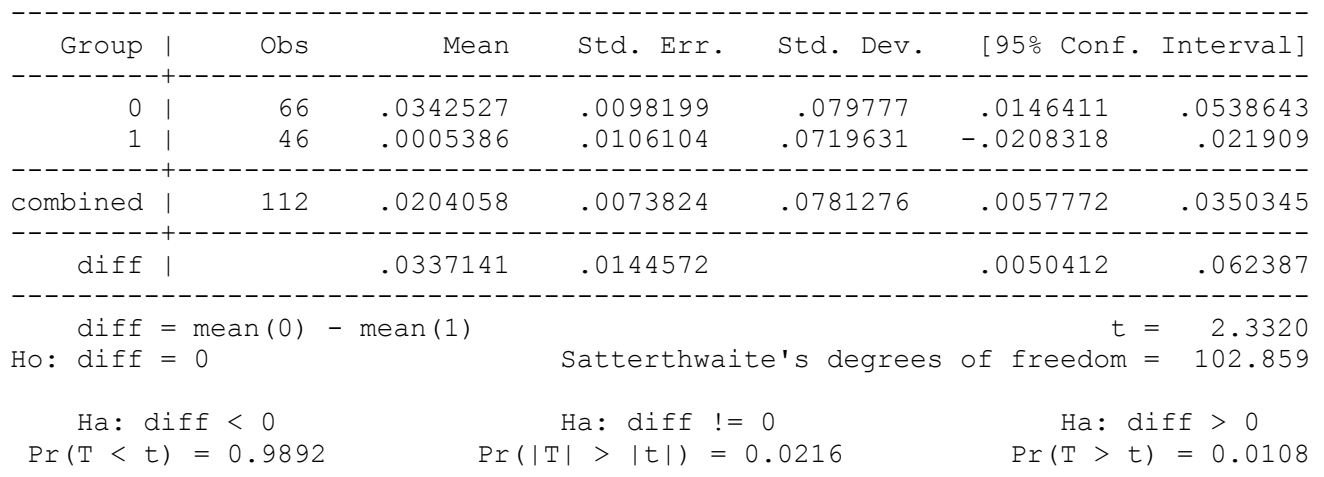

- ttest alpha, by (female) unequal

Two-sample $t$ test with unequal variances

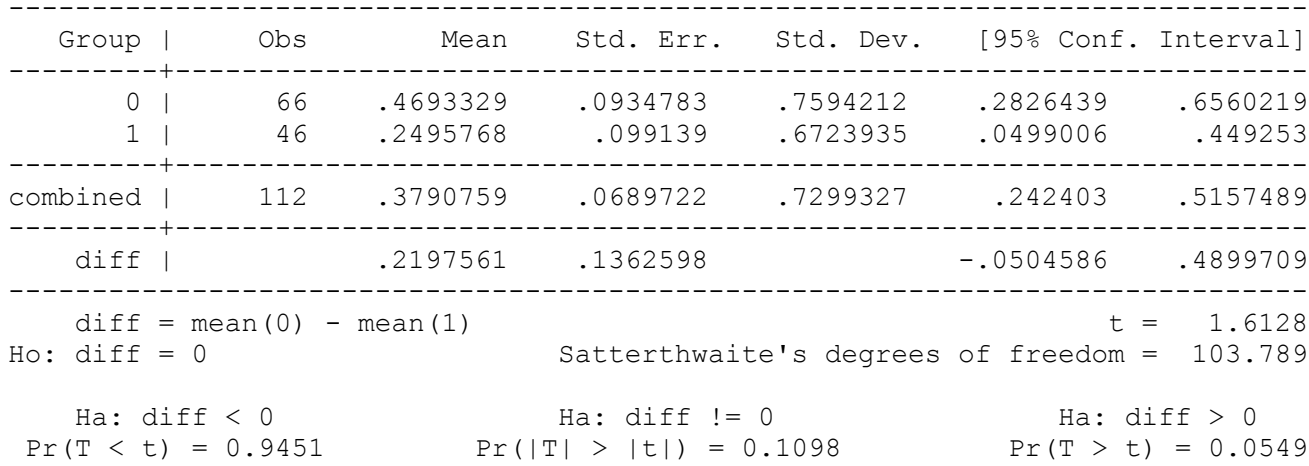

